\title{
MECP2 promotes migration and invasion of gastric cancer cells via modulating the Notch1/c- Myc/mTOR signaling pathways by suppressing FBXW7 transcription
}

\section{Lingyu Zhao}

Xi'an Jiaotong University School of Medicine https://orcid.org/0000-0001-9616-2183

\section{Xiaofei Wang}

Xi'an Jiaotong University School of Medicine

Juan Yang

Xi'an Jiaotong University School of Medicine

Qiuyu Jiang

Xi'an Jiaotong University School of Medicine Jing Zhang

Medical College of Yan'an University

Yannan Qin

Xi'an Jiaotong University School of Medicine

\section{Lumin Wang}

Xi'an Jiaotong University School of Medicine

Liying Liu

Xi'an Jiaotong University School of Medicine

Lei Ni

Xi'an Jiaotong University School of Medicine

Dongdong Tong ( $\sim$ tongdd@xitu.edu.cn )

Xi'an Jiaotong University School of Medicine

Chen Huang

\section{Research}

Keywords: MECP2, Gastric cancer, FBXW7, migration, invasion, Notch1 signaling pathway

Posted Date: July 28th, 2020

DOI: https://doi.org/10.21203/rs.3.rs-48700/v1 
License: (c) (i) This work is licensed under a Creative Commons Attribution 4.0 International License. Read Full License 


\section{Abstract}

Background: Methyl-CpG-binding protein 2 (MECP2), an epigenetic regulatory factor, promotes the carcinogenesis and progression of a number of cancers. However, its role in the migration and invasion of gastric cancer (GC) as well as the underlying molecular mechanisms remain unclear.

Methods: Immunohistochemistry (IHC), Western blot, and quantitative real-time PCR (qRT-PCR) were performed to measure the expressions of MECP2, FBXW7, c-Myc, mTOR and Notch1 in GC tissues and cell lines, respectively. The effects of MECP2 silencing and overexpression on GC cell migration and invasion were detected by wound healing assay and transwell assay. The mechanisms of MECP2mediated migration and invasion were further investigated using chromatin immunoprecipitation sequencing (ChIP-Seq) and luciferase reporter gene assay.

Results: In this study, we found that MECP2 facilitated the migration and invasion of GC cells. Investigation of the molecular mechanism revealed that MECP2 restrained FBXW7 transcription in GC by binding to the methylated $\mathrm{CpG}$ sites of FBXW7's promoter region. MECP2 expression was remarkably negatively correlated with the FBXW7 level in GC tissues. FBXW7 expression was significantly downregulated in GC tissues and cell lines, and low FBXW7 expression was correlated with the clinicopathologic features. FBXW7 repressed cell migration and invasion by regulating the Notch1/c$\mathrm{Myc} / \mathrm{mTOR}$ signaling pathways, and knockdown of FBXW7 reversed the effects of silencing MECP2. Moreover, MECP2 upregulated the Notch1/c-Myc/mTOR signaling pathways by inhibiting FBXW7 expression at the transcription level.

Conclusion: This study demonstrates that MECP2 promotes migration and invasion of GC cells via modulating the Notch1/c-Myc/mTOR signaling pathways by suppressing FBXW7 transcription. The findings suggest that MECP2 may be a novel effective therapeutic target for GC patients.

\section{Background}

Gastric cancer (GC) remains the fifth most common malignant cancer and the third most frequent cause of cancer-related deaths worldwide, and the highest mobidity and mortality are seen in East Asia [1, 2]. Although the incidence of GC has reduced evidently in the last few decades, there remain approximately 679,000 new GC cases and 498,000 GC cancer-associated deaths in China every year [3]. At present, GC patients are usually treated with a combination of surgery, chemotherapy and radiotherapy, but the effect is unsatisfactory because it is a highly complex disease. Especially, the molecular mechanisms of GC metastasis and invasion, which is responsible for nearly $90 \%$ of the lethality among GC patients, are still not fully understood [4-6]. Therefore, it is crucial to investigate genes that govern GC metastasis and invasion so as to illuminate the molecular mechanisms and develop more effective therapeutic strategies.

Methyl-CpG-binding protein 2 (MECP2), belonging to the methyl-CpG-binding domain (MBD) family, contains a MBD and a transcriptional repression domain (TRD) [7-9]. As an important epigenetic 
regulatory factor, MECP2 modulates chromatin organization and gene transcription by binding to the methylated DNA sites of promoter regions of genes $[10,11]$. It is an X-linked gene whose mutation results in neurological disorders, such as Rett syndrome [12]. It has been reported that MECP2 not only suppresses gene transcription, such as inhibiting BDNF and Cdkl5 expressions, via binding to the methylated $\mathrm{CpG}$ islands and recruiting co-repressors (e.g., histone deacetylases and $\operatorname{Sin} 3 \mathrm{~A}$ ), but also promotes gene transcription, such as facilitating GIT1 expression, through binding to the methylated CpG dinucleotides and recruiting activators (e.g., CREB1) [13-17]. In recent years, emerging evidence indicates the role of MECP2 as a crucial oncogene in several cancer types [18]. Our previous studies have found that MECP2 is upregulated in liver cancer and facilitates cell growth [19], it enhances breast cancer proliferation via facilitating ubiquitination-mediated P53 degradation by regulating RPL5/RPL11 expressions [20], and it promotes GC cell proliferation and suppresses cell apoptosis via restraining FOXF1/MYOD1 transcription and enhancing GIT1 transcription by binding to the methylated $\mathrm{CpG}$ sites of the promoter regions of FOXF1/MYOD1 [21, 22]. Cancer cell migration and invasion are related to the metastatic potential of the cells, which may be independent of cell proliferation rates. By far, the function of MECP2 in cancer cell migration and invasion has not been precisely studied. In particular, the role of MECP2 in the migration and invasion of GC remains unknown.

In response to the above mentioned gap, we investigated the effect and the molecular mechanism of MECP2 on GC cell migration and invasion. The results demonstrated that MECP2 facilitated the migration and invasion of GC cells. Chromatin immunoprecipitation sequencing (ChIP-Seq) and luciferase reporter gene assay showed that MECP2 inhibited F-box and WD40 domain protein 7 (FBXW7) transcription by binding to the methylated $\mathrm{CpG}$ site of FBXW7's promoter region. Western blot and qRTPCR revealed a lower expression of FBXW7 in GC tissues, which was correlated with the clinicopathologic features. MECP2 expression was negatively correlated with FBXW7 expression. FBXW7 restrained the migration and invasion of GC cells. Further molecular mechanistic investigations found that MECP2 promoted cell migration and invasion via regulating the Notch1/c-Myc/mTOR signaling pathways by inhibiting FBXW7 transcription. In brief, the findings in this study indicate that MECP2 may be a novel effective therapeutic target for GC.

\section{Methods}

\section{Patients and specimens}

Tumor specimens and adjacent nontumor tissues were collected from 166 GC patients treated from 2015 to 2018 in the Department of Oncology Surgery, the First Affiliated Hospital of Medical College in Xi'an Jiaotong University, PR China. All the patients underwent curative surgical resection without radiotherapy or chemotherapy prior to surgery. Clinicopathological data of the patients were obtained from their pathology records. The study was approved by the Ethical Committee of the university. Informed consents were obtained from all the patients before tissue sample collection.

\section{Cell culture}


Normal human gastric epithelial cell line (GES-1) and human GC cell lines BGC-823 and MKN-45 were purchased from the Cell Bank (Shanghai Genechem Co., Ltd., Shanghai, China). The cell lines had been tested and authenticated by the Cell Bank. Cells were cultured in RPMI-1640 medium (Gibco BRL, Grand Island,NY, USA) containing $10 \%$ fetal bovine serum (FBS) (Gibco, USA) at $37^{\circ} \mathrm{C}$ in $5 \% \mathrm{CO}_{2}$ and $100 \%$ humidity. All the cells were examined and validated negative for mycoplasma contamination. Cell morphology and behavior were confirmed consistent with the Cell Bank descriptions.

\section{RNA extraction and quantitative real-time PCR (qRT-PCR)}

Total RNA was isolated from GC tissues and cell lines using TRIzol Reagent (Invitrogen, Carlsbad, CA, USA) according to the manufacturer's protocol. Reverse transcription was fulfilled using a commercial kit (Takara, Dalian, China). qRT-PCR was performed with the SYBR Green PCR kit (Takara Biotechnology, Takara, Dalian, China) using an IQ5 Multicolor qRT-PCR Detection System (Bio-Rad, USA). All qRT-PCR reactions were implemented in triplicates for each specimen. The primer sequences were listed in Additional file 1: Table S1. The mRNA expressions of relative genes were normalized to $\beta$-Actin and the $2^{-\Delta \Delta C t}$ method was used to analyze the expression levels.

\section{Immunohistochemical (IHC) staining}

IHC was performed on the tissue specimens from GC patients. The tissue samples were fixed in $4 \%$ paraformaldehyde, embedded in paraffin and sectioned at a thickness of $4 \mu \mathrm{m}$. After deparaffinization and hydration with graded alcohol, antigen retrieval and blocking were performed. The sections were then incubated with corresponding primary antibody (anti-FBXW7, Santa Cruz, CA, USA) at a dilution of 1: 100, followed by incubation with biotinylated secondary antibody (Santa Cruz, CA, USA). Subsequently, the sections were detected using the 3, 3'-diaminobenzidine (DAB) kit and hematoxylin, and were scored by two pathologists independently without knowing the patient outcome. FBXW7 protein expression was assessed semi-quantitatively. High expression was defined as the proportion of positive cells being $>25 \%$ in 5 random fields; otherwise, the expression was considered low. The integral optical density (ISO) in 5 randomly selected fields was quantified using ImageJ v1.8.0 (Wayne Rasband, USA).

\section{Plasmid construction and transfection}

Human full-length MECP2 and FBXW7 complementary DNAs were cloned respectively into the pCMV2GV146 vector following the manufacturer's instructions (Genechem Co. Ltd, China). Then, the wild type and mutation type pasmids, pCMV2-GV146-GFP-MECP2 plasmid (WT), pCMV2-GV146-GFP-Mutation type 1 plasmid (MT1), and pCMV2-GV146-GFP-Mutation type 2 plasmid (MT2) were constructed (Additional file 1: Table S2). The reporter plasmid pGL3-FBXW7 containing a 463-bp fragment spanning from 153454019 to 153454481 relative to the transcription start site (TSS) of FBXW7 promoter, was placed upstream of the firefly luciferase reporter gene (pGL3-FBXW7-luc) (Genechem Co. Ltd., Shanghai, China). BGC-823 and MKN-45 cells were cultured in RPMI-1640 medium without antibiotics for $24 \mathrm{~h}$. Next, pCMV2-GV146 vector, pCMV2-GV146-MECP2 plasmid or PCMV2-GV146-FBXW7 plasmid was transiently transfected into the cells using Lipofectamine TM-2000 reagent (Invitrogen, Carlsbad, CA, USA). Cell assays were performed $48 \mathrm{~h}$ after transfection. 


\section{siRNA synthesis and transfection}

The sequences of MECP2 siRNAs (si-MECP2-1 and si-MECP2-2) and FBXW7 siRNAs (si-FBXW7-1 and siFBXW7-2) were pre-designed and synthesized by GenePharma (SGC, Shanghai, China). Scramble siRNA was used as negative control (NC-siRNA). All the siRNA sequences were listed in Additional file 1: Table S3. GC cells (BGC-823 and MKN-45) were cultured for $24 \mathrm{~h}$ and transfected with indicated siRNAs or NCsiRNA using Lipofectamine TM-2000 reagent in accordance with the manufacturer's instructions.

\section{Immunofluorescence (IF)}

BGC-823 and MKN-45 cells were plated on the polylysine-coated coverslips in 24-well plates for $24 \mathrm{~h}$ and then transfected with pCMV2-GV146-GFP plasmid or pCMV2-GV146-GFP-MECP2 plasmid for $48 \mathrm{~h}$. Following that, the cells were fixed in $4 \%$ paraformaldehyde at room temperature for $10 \mathrm{~min}$, washed with PBS, and blocked with 10\% normal goat serum in PBS containing $0.3 \%$ Triton $\mathrm{X}-100$ for $1 \mathrm{~h}$ at room temperature. Subsequently, the cells were incubated at $4{ }^{\circ} \mathrm{C}$ with anti-GFP or anti-MECP2 primary antibody (Additional file 1: Table S4) overnight. Negative controls lacking the primary antibodies were used for eliminating nonspecific staining. Subsequently, the matched secondary antibodies IgG-FITC and IgG-PE were added, followed by $2 \mathrm{~h}$ of incubation at $37^{\circ} \mathrm{C}$, after which 4, 6-diamidino-2-phenylindole (DAPI) was used to stain cell nuclei for $5 \mathrm{~min}$. The immunostained cells were observed by indirect fluorescence under a fluorescent microscope (Olympus BX51; Olympus, Tokyo, Japan) equipped with the DPManager (DPController) software (Olympus). Each expeiment was conducted independently three times.

\section{Wound healing assay}

The scratch wound healing assay was performed to detect the migration capacity of human GC cells. Differently treated BGC-823 and MKN-45 cells were seeded in 6-well plates and reached approximately $60 \%$ confluence. A single wound was gently and slowly scratched with a $10 \mu \mathrm{l}$ disposable pipette tip across the center of a well. Suspension cells were removed by washing with PBS, and the remaining cells were incubated in fresh medium. The wound closures were observed at 0 and $48 \mathrm{~h}$ under a microscope (Olympus, Japan). The gap distance was quantitatively evaluated using ImageJ v1.8.0 (Wayne Rasband, USA). The migration rate of cells was quantified by setting average migration distance in control group as $100 \%$. Results were obtained from all the three independent experiments.

\section{Transwell migration and invasion assays}

Transwell assays were performed on BGC-823 and MKN-45 cells. For examining cell migration, differently treated cells were planted in the upper chambers of the 24-well transwell (8- $\mu \mathrm{m}$ pore size; Millipore, Billerica, MA, USA) at a density of $2.0 \times 10^{4}$ cells in $100 \mu \mathrm{l}$ serumfree medium, and $600 \mu \mathrm{l}$ of $10 \%$ serumcontaining medium was added to the lower chambers. Cells were then incubated for $24 \mathrm{~h}$ at $37^{\circ} \mathrm{C}$ in a $5 \%$ $\mathrm{CO}_{2}$ atmosphere and then removed from the upper surface of the filter by scraping with a cotton swab. The migrated cells adhering to the bottom membrane were fixed with $4 \%$ paraformaldehyde and stained with $0.1 \%$ crystal violet. For examining cell invasion, $4.0 \times 10^{4}$ cells were maintained in the matrigel 
(15 $\mu \mathrm{g} /$ filter; BD Biosciences, Franklin Lakes, NJ, USA) coated chamber for $48 \mathrm{~h}$. Cells that had migrated and invaded to the lower surface of the filter were figured with ImageJ v1.8.0 (Wayne Rasband, USA) in 5 randomly fields. The relative migration and invasion rates were measured by setting the number of cells in the control groups as 1. Each experiment was repeated three times.

\section{Chromatin Immunoprecipitation (ChIP), ChIP-Seq and ChIP- qRT-PCR}

Different plasmids, including pCMV2-GV146-GFP-MECP2 plasmid (WT), pCMV2-GV146-GFP-Mutation type 1 plasmid (MT1), pCMV2-GV146-GFP-Mutation type 2 plasmid (MT2) and empty plasmid, were transfected into BGC-823 cells. ChIP was performed as previously described [23]. The cultured BGC-823 cells were crosslinked with 1\% formaldehyde for $15 \mathrm{~min}$, and $125 \mathrm{mM}$ glycine was added for quenching. A cell cracker was used to sonicate cells to obtain nuclear lysates. The chromatin was sonicated into approximate 200-bp DNA fragments. The obtained lysates were divided into two portions. One portion was used as input, and the other was incubated with $5 \mu \mathrm{g}$ antibodies against GFP, MeCP2, or IgG (Additional file 1: Table S4) overnight at $4{ }^{\circ} \mathrm{C}$. Next, DNA-protein complexes were captured with Dynabeads Protein A (Invitrogen, Carlsbad, CA, USA) and eluted with TE buffer at $65^{\circ} \mathrm{C}$. The crosslinking of DNA-protein complexes was reversed for $8 \mathrm{~h}$ at $65^{\circ} \mathrm{C}$. DNA was extracted with the QIAquick PCR purification kit (QIAGEN, Hilden, Germany). The sequencing of DNA was performed on an Illumina HiSeq 2000 using TruSeq Rapid SBS Kits (Illumina, San Diego, CA, USA, FC-402-4002) in accordance with the manufacturer's protocols. ChIP-enriched DNA fragments were located based on the Human Feb 2009 assembly and visualized using the genome browser of the University of California. Peak calling in the mapped ChIP-Seq data was fulfilled with ChIP-Peak and subjected to further bioinformatics analysis. The ChIP-Seq experiments were performed by KangChen Biotech (KangChen, Shanghai, China). The genespecific primers were pre-designed and synthesized for analyzing the captured DNA fragments via qRTPCR or RT-PCR. The primer sequences were as follows: FBXW7 forward primer (5'-

GACTCTGTCTCAAAAAAAAAAAAAA-3') and FBXW7 reverse primer (5'-GAGGACAAACTAAGAAACAAACTAT$\left.3^{\prime}\right)$.

\section{FBXW7-Luciferase reporter gene assay}

BGC-823 cells were planted into 96-well plates, five wells per group. pGL3-FBXW7-luc plasmid was amplified in DH5a and treated with CpG methyltransferase M.SssI (M0226S, NEB, USA) for $48 \mathrm{~h}$ to form the pGL3-FBXW7-luc + Methylation plasmid. The cells were then transfected with pGL3-luc, pGL3-FBXW7luc, and pGL3-FBXW7-luc + Methylation plasmids, respectively, for $48 \mathrm{~h}$. In a separate expeiment, the cells were first transfected with pGL3-FBXW7-luc or pGL3-FBXW7-luc + Methylation plasmid, and then with NCsiRNA, si-MECP2-1, si-MECP2-2, control plasmid vector, MECP2-overexpressing plasmid, dimethyl sulphoxide (DMSO), and methylation inhibitor 5-aza-2'-deoxycytidine (Aza), respectively. Luciferase activity was examined at $48 \mathrm{~h}$ post-transfection with a Dual-Luciferase Reporter Assay System (Promega, Madison, WI). Each experiment was performed indepently three times.

\section{Western blot analysis}


The total protein from human GC cell lines and tissues were lysed for whole-cell extracts with RIPA lysis buffer (Cell Signaling Technology, Boston, MA) complemented with protease inhibitors (Roche, Indianapolis, IN, USA). Nuclear proteins were extracted using a Nuclear and Cytoplasmic Extraction Kit (Thermo Fisher Scientific, Waltham, MA, USA) and a CelLytic ${ }^{\text {TM }}$ NuCLEAR $^{\text {TM }}$ Extraction Kit (Sigma, St. Louis, MO, USA) according to the manufacturers' instructions. Protein concentrations of cellular or nuclear extracts were measured using a BCA Assay Kit (Thermo Fisher Scientifc, Waltham, MA, USA). Protein extracts $(25 \mu \mathrm{g})$ were run on $10 \%$ sodium dodecyl sulfate-polyacrylamide gel electrophoresis and transferred to PVDF membranes. The membranes were incubated with primary antibodies at $4{ }^{\circ} \mathrm{C}$ overnight and then with the corresponding secondary antibodies for $1 \mathrm{~h}$ at room temperature. After that, ECL (Pierce, Rockford, IL, USA) was used to incubate the membranes for chemiluminescence detection. The luminescent signal was measured and recorded with a Syngene Gbox (Syngene, Cambridge, UK). $\beta$ Actin expression was used as the loading control. The primary antibodies used are listed in Additional file 1: Table S4.

\section{Statistical analysis}

All data were analyzed using Graphpad 7.0 (Graph-Pad Software, USA) and SPSS 19.0 (Abbott Laboratories, Chicago, IL, USA). Results are expressed as Mean \pm SEM from at least 3 independent experiments. Student's t-test was used to compare two independent groups. One-way ANOVA followed by individual comparisons using Dunnett's test was performed to analyze differences among more than two groups. Chi-square test was employed to detect the relationships between FBXW7 expression and clinicopathologic characteristics. Pearson's correlation analyses were conducted to estimate the association of MECP2 with FBXW7. $P<0.05$ was considered statistically significant.

\section{Results}

\section{MECP2 is overexpressed in GC and closely related with the clinicopathological characteristics}

Analysis based on the Cancer Genome Atlas (TCGA) data identified significant overexpression of MECP2 in GC tissues ( $P<0.001$, Fig. 1a). qRT-PCR on the GC tissue samples and paired adjacent normal gastric tissue samples from $166 \mathrm{GC}$ cases collected in this study revealed a remarkably upregulated MECP2 mRNA expression in cancer tissues $(P<0.001$, Fig. 1b). This result is consistent with that of our previous study which was based on 76 GC patients [21]. Further analysis suggested a correlation between MECP2 expression and clinicopathologic characteristics. TCGA data also revealed an association of high MECP2 expression with tumor histology and T stage $(P<0.01$, Fig. 1c, d).

\section{MECP2 facilitates the migration and invasion of GC cells}

To investigate the causal role of MECP2 in GC progression, a MECP2-overexpressing plasmid was constructed and MECP2-targeting siRNAs (si-MECP2-1 and si-MECP2-2) were designed and synthesized. 
qRT-PCR showed that MECP2-overexpressing plasmid observably upregulated the mRNA expression of MeCP2 in BGC-823 and MKN-45 cells ( $P<0.001$, Fig. 2a), while si-MECP2-1 and si-MECP2-2 significantly downregulated the MeCP2 mRNA level $(P<0.001$, Fig. 2b). Immunofluorescent assay revealed that a higher level of exogenous MECP2 protein in the GFP-MECP2-vector group than in the GFP-vector group in both BGC-823 and MKN-45 cells (Additional file 2: Figure S1A, B). Our previous study demonstrated that MECP2 promoted cell proliferation and inhibited cell apoptosis in GC [21,22]. To further study the effect of MECP2 on GC progression, a wound-healing assay and transwell assays were performed to explore the effect of MECP2 on the migration and invasion ability of GC cells. The wound-healing assay showed that MECP2-overexpressing plasmid significantly enhanced the migration of the cells into the scratched area in GC BGC-823 and MKN-45 cells, while si-MECP2-1 and si-MECP2-2 markedly suppressed cell migration $(P<0.01$, Fig. 2c, d). Consistently, transwell assays also revealed a promoting effect of MECP2overexpressing plasmid and an inhibitive effect of si-MECP2-1 and si-MECP2-2 on cell migration $(P<0.01$, Fig. 2e, f). Meanwhile, MECP2-overexpressing plasmid remarkably facilitated cell invasion, and si-MECP21 and si-MECP2-2 significantly restrained cell invasion $(P<0.01$, Fig. $2 \mathrm{~g}, \mathrm{~h})$. Further exploration of the molecular mechanism of MECP2 modulation in GC progression revealed that the protein expressions of MECP2, MMP-2 and MMP-9 were upregulated by MECP2-overexpressing plasmid but downregulated by si-MECP2-1 and si-MECP2-2 (Fig. 2i, j). These data suggested that MECP2 might promote the migration and invasion of GC cells through regulating MMP-2 and MMP-9 expressions.

\section{MECP2 suppresses FBXW7 transcription by binding to its promoter}

To explore how MECP2 might regulate GC cell migration and invasion, ChIP-Seq assay was performed in BGC-823 cells to identify the genes regulated by MECP2. Altogether 8128 ChIP-Seq peaks with various fold enrichments were acquired, among which 220 peaks were located in the promoter regions of genes (Additional file 3: Table S5). Then, MethPrimer was used to predict potential CpG sites within the MECP2 binding region. The results showed that the MECP2 binding region in the promoter region of FBXW7 contained a CpG site (Cg01181485, Fig. 3a). ChIP RT-PCR also verified that MECP2 could directly bind to the promoter of FBXW7 (Fig. 3b). The wild type (WT) and mutation type (MT) GFP-MECP2 plasmids were constructed. After they were transfected into BGC-823 cells, we fulfilled ChIP RT-PCR with anti-GFP antibody. The finding revealed that exogenous MECP2 could also bind to the CpG site of FBXW7 promoter region (Fig. 3c). GFP plasmid (Ctrl), GFP-MT1 and GFP-MT2 could not bind to the site, but GFPWT could bind to it (Fig. 3d). TCGA data showed that the methylation level of the CpG site (Cg01181485) was inversely correlated with the expression of FBXW7 in GC $(P<0.01$, Fig. 3e).

Subsequently, a promoter reporter assay was fulfilled to determine whether MECP2 could bind to the CpG site of the promoter region of FBXW7. The binding sequences of FBXW7's promoter region from ChIP-Seq were subcloned into the upstream of the luciferase gene in the pGL3 reporter plasmid (Additional file 1: Table S5). The luciferase activity was detected at $48 \mathrm{~h}$ after transfection with different plasmids in BGC823 cells. It was found that the luciferase activity was evidently reduced in the pGL3-FBXW7-luc and 
pGL3-FBXW7-luc + Methylation groups as against the pGL3 group, and the luciferase activity in the pGL3FBXW7-luc + Methylation group was significantly lower than in the pGL3-FBXW7-luc group $(P<0.01$, Fig. 3f).

Among the groups of transfection first with pGL3-FBXW7-luc plasmid and then with NC-siRNA, si-MECP21, si-MECP2-2, control plasmid vector, MECP2-overexpressing plasmid, dimethyl sulphoxide (DMSO), and methylation inhibitor 5-aza-2'-deoxycytidine (Aza), respectively, the luciferase activity was observably enhanced in the si-MECP2-1 and si-MECP2-2 groups as compared with in the NC-siRNA group, remarkably increased in the methylation inhibitor 5-aza-2'-deoxycytidine (Aza) group as against the DMSO group, and significantly decreased in the MECP2-overexpressing plasmid group as against the vector group. The groups of first transfection with pGL3-FBXW7-luc + Methylation plasmid and then the further treatment generated similar results, but the activity was lower than that in the pGL3-FBXW7-luc groups $(P<0.01$, Fig. $3 g$ ). The MECP2 mRNA expression was significantly negatively correlated with the FBXW7 mRNA level in GC tissues ( $r=0.6302, P<0.0001$, Pearson's correlation, Fig. 3h). Furthermore, the FBXW7 mRNA expression in BGC-823 and MKN-45 cells remarkably decreased after transfection with MECP2overexpressing plasmid and increased after treatment with the methylation inhibitor Aza, si-MECP2-1 and si-MECP2-2 (Additional file 4: Figure S2A-C). The protein expression of FBXW7 in the cells was also inhibited by MECP2-overexpressing plasmid (Fig. 3i), but facilitated by the methylation inhibitor Aza, siMECP2-1 and si-MECP2-2 (Fig. 3j, k). These findings suggested MECP2 as a transcription regulator of FBXW7 in GC cells.

\section{FBXW7 is frequently downregulated in human GC tissues and is correlated with the clinicopathologic features}

To further verify the findings, FBXW7 expression in the tissue samples was measured. TCGA data revealed a remarkable downregulation of FBXW7 expression in GC tissues as compared with in normal gastric tissues $(P<0.001$, Fig. $4 a)$. In line with this, our statistical analyses showed that patients with lower FBXW7 levels had poorer overall survival $(P<0.05$, Fig. 4b). The mRNA expression of FBXW7 was significantly downregulated in GC tissues $(P<0.0001$, Fig. 4C). This trend was further verified by measuring the FBXW7 expression in GC BGC-823 and MKN-45cells. The mRNA expression of FBXW7 in BGC-823 and MKN-45 cells was evidently lower than in normal human gastric epithelial cells (GES-1) $(P<$ 0.001 , Fig. 4d). The protein expression of FBXW7 was markably lower in GC tissues than in normal gastric tissues (Fig. 4e). Our data showed that FBXW7 expression and clinicopathologic characteristics of GC patients were corelated. Low FBXW7 expression was associated with tumor size ( $\geq 50 \mathrm{~mm}: 86.5 \%$; < 50 mm: 57.1\%), lymph node metastasis (Absent: 39.7\%; Present: 90.7\%), lymphatic invasion (Absent: 46.3\%; Present: 85.7\%), venous invasion (Absent: 65.6\%; Present: 82.2\%), serosal invasion (Absent: 50.9\%; Present: 84.4\%), TNM stage (I: 48.4\%; II: 67.4\%; III: 82.5\%; IV: 87.5\%) (Table 1). The expression was not associated with age, gender, histology, and liver metastasis. Western blot also detected decrease of FBXW7 protein expression in 5 pairs of GC and ajacent normal tissues, and GC BGC-823 and MKN-45 cells (Fig. 4f, g). 
Table 1

Patient characteristics and clinicopathologic correlation of FBXW7 expression in GC

\begin{tabular}{|c|c|c|c|c|c|}
\hline \multirow[t]{2}{*}{ Characteristics } & \multirow[t]{2}{*}{ Number of cases } & \multicolumn{3}{|c|}{ FBXW7 protein expression } & \multirow[t]{2}{*}{ P-value } \\
\hline & & & $=45)$ & $\operatorname{Low}(n=121)$ & \\
\hline Age & & & & & 0.736 \\
\hline$\geq 60$ years & 75 & 18 & 57 & & \\
\hline$<60$ years & 91 & 27 & 64 & & \\
\hline Gender & & & & & 0.315 \\
\hline Male & 102 & 24 & 78 & & \\
\hline Female & 64 & 21 & 43 & & \\
\hline Histology & & & & & 0.382 \\
\hline Well & 86 & 21 & 65 & & \\
\hline Moderate & 32 & 13 & 19 & & \\
\hline poor & 48 & 11 & 37 & & \\
\hline
\end{tabular}




\begin{tabular}{|c|c|c|c|c|c|}
\hline \multirow{3}{*}{$\begin{array}{l}\text { Characteristics } \\
\text { Size }\end{array}$} & \multirow{3}{*}{$\begin{array}{l}\text { Number of cases } \\
77\end{array}$} & \multicolumn{3}{|c|}{ FBXW7 protein expression } & \multirow{3}{*}{$\begin{array}{l}\text { P-value } \\
0.0005^{*}\end{array}$} \\
\hline & & \multicolumn{2}{|c|}{ High $(n=45)$} & \multirow[t]{2}{*}{ Low $(n=121)$} & \\
\hline & & 33 & 44 & & \\
\hline$<50 \mathrm{~mm}$ & 89 & 12 & 77 & & $0.0001 *$ \\
\hline$\geq 50 \mathrm{~mm}$ & 58 & 35 & 23 & & $0.0008^{*}$ \\
\hline Lymph node metastasis & 108 & 10 & 98 & & $0.031^{\star}$ \\
\hline Absent & 54 & 29 & 25 & & $0.0007^{*}$ \\
\hline Present & 112 & 16 & 96 & & 0.208 \\
\hline Lymphatic invasion & 93 & 32 & 61 & & \\
\hline Absent & 73 & 13 & 60 & & \\
\hline Present & 57 & 28 & 29 & & \\
\hline Venous invasion & 109 & 17 & 92 & & \\
\hline Absent & 124 & 37 & 87 & & \\
\hline Present & 42 & 8 & 34 & & \\
\hline \multicolumn{6}{|l|}{ Serosal invasion } \\
\hline \multicolumn{6}{|l|}{ Absent } \\
\hline \multicolumn{6}{|l|}{ Present } \\
\hline \multicolumn{6}{|l|}{ Liver metastasis } \\
\hline \multicolumn{6}{|l|}{ Absent } \\
\hline \multicolumn{6}{|l|}{ Present } \\
\hline TNM Stage & & & & & $0.0024^{\star}$ \\
\hline I & 31 & 16 & 15 & & \\
\hline II & 46 & 15 & 31 & & \\
\hline III & 57 & 10 & 47 & & \\
\hline IV & 32 & 4 & 28 & & \\
\hline
\end{tabular}

\section{MECP2 upregulates the Notch1/c-Myc/mTOR signaling pathways by repressing FBXW7 transcription}

To investigate the potential molecular mechanisms of MECP2-regulated GC cell migration and invasion, mRNA and protein levels of the FBXW7-related downstream genes were measured by qRT-PCR and Western blot. FBXW7 is a substrate recognition subunit of the SKP1-CUL1-F-box protein (SCF) E3 
ubiquitin ligase complex, which plays a crucial role in tumorigenesis and cancer progression by promoting degradation of ubiquitination-mediated oncoproteins, including mTOR, c-Myc, c-Jun, and Notch1 [24-26]. The results of qRT-PCR showed that the mRNA expressions of Hes1, MMP-2 and MMP-9 significantly increased in BGC-823 and MKN-45 cells after transfection with MECP2-overexpressing plasmid, and they dramatically decreased after the treatment with the methylation inhibitor Aza, siMECP2-1, and si-MECP2-2 $(P<0.001$, Fig. 5a-c). However, there were no significant differences in the mRNA levels of c-Myc, mTOR and Notch1 after the treatment (Additional file 5: Figure S3A-C). The Western blot analysis revealed that the protein expressions of c-Myc, mTOR, Notch1, Hes1, MMP-2 and MMP-9 were upregulated in whole-cells after transfection with MECP2-overexpressing plasmid, and they were downregulated after the treatment with methylation inhibitor Aza, si-MECP2-1 and si-MECP2-2 (Fig. 2i, j; Fig. 5d-f). In addition, the change of nuclear NICD1 protein level was consistent with the change of whole-cell Notch1 protein level (Fig. 5d-f). Based on these findings, MECP2 might regulate the Notch1/c-Myc/mTOR signaling pathways via inhibiting degradation of ubiquitination-mediated Notch1, c-Myc and mTOR by suppressing FBXW7 transcription in GC.

\section{FBXW7 restrains GC cell migration and invasion by regulating the Notch1/c-Myc/mTOR signaling pathways}

Since MECP2 regulated GC cell migration and invasion, and FBXW7 was confirmed as a target gene of MECP2 modulation, FBXW7 overexpression and knock-down were performed in the GC cell lines to verify its involvement in the oncogene function of MECP2. The wound-healing assay revealed that the FBXW7overexpressing plasmid substantially repressed the migration of cells into the scratched area in GC BGC823 and MKN-45 cells, but si-FBXW7-1 and si-FBXW7-2 significantly promoted GC cell migration $(P<0.01$, Fig. 6a, b). The transwell assays also showed that FBXW7-overexpressing plasmid markedly suppressed GC cell migration, and si-FBXW7-1 and si-FBXW7-2 strikingly facilitated GC cell migration $(P<0.01$, Fig. 6c, d). Moreover, the FBXW7-overexpressing plasmid significantly inhibited cell invasion, while siFBXW7-1 and si-FBXW7-2 remarkably enhanced GC cell invasion $(P<0.01$, Fig. 6e, f). Then, the downstream mechanism of FBXW7 regulation was further explored. qRT-PCR showed that the FBXW7overexpressing plasmid evidently increased the FBXW7 mRNA expression in BGC-823 and MKN-45 cells $(P<0.001$, Fig. 6g), while si-FBXW7-1 and si-FBXW7-2 remarkably decreased the expression $(P<0.001$, Fig. 6h). No significant differences were observed in the levels of c-Myc, mTOR and Notch1 after transfection with FBXW7-overexpressing plasmid, si-FBXW7-1 and si-FBXW7-2 (Additional file 6: Figure S4A-C). The mRNA levels of Hes1, MMP-2 and MMP-9 in the cells remarkably decreased after transfection with the FBXW7-overexpressing plasmid, and obviously increased after transfection with siFBXW7-1 and si-FBXW7-2 ( $P<0.001$, Additional file 6: Figure S4D-F). Western blot showed that the FBXW7-overexpressing plasmid upregulated FBXW7 protein expression, while si-FBXW7-1 and si-FBXW72 downregulated the expression. Meanwhile, the protein expressions of c-Myc, mTOR, Notch1, Hes1, MMP-2 and MMP-9 in whole-cells were reduced after transfection with FBXW7-overexpressing plasmid but enhanced after transfection with si-FBXW7-1 and si-FBXW7-2. The change of nuclear NICD1 protein level was consistent with that of the whole-cell Notch1 protein level (Fig. 6i, j). 


\section{Knockdown of FBXW7 reverses the effect of silencing MECP2 on GC cells}

To further confirm that MECP2 facilitates GC cell migration and invasion by repressing FBXW7 expression, FBXW7 siRNA was co-transfected with MECP2 siRNA into BGC-823 or MKN-45 cells. The wound-healing assay showed that silencing MECP2 inhibited GC cell migration, and this effect was eliminated by knockdown of FBXW7 $(P<0.01$, Fig. 7a). The transwell assays also revealed that silencing MECP2 suppressed cell migration, which was reversed after co-transfection with MECP2 siRNA and FBXW7 siRNA ( $P<0.01$, Fig. 7b). In addition, co-transfection with MECP2 siRNA and FBXW7 siRNA rescued the effect of MECP2 knockdown on cell invasion ( $P<0.01$, Fig. 7C).

The downstream regulators involved in the promotion of cell migration and invasion by MECP2 were determined by qRT-PCR. MECP2 mRNA expression significantly decreased in BGC-823 and MKN-45 cells after transfection with MECP2 siRNA or MECP2 siRNA + FBXW7 siRNA $(P<0.01$, Fig. 7d). The mRNA expression of FBXW7 markedly increased after transfection with MECP2 siRNA, and this effect was eliminated by transfection with MECP2 siRNA + FBXW7 siRNA $(P<0.01$, Fig. 7e). There were no significant differences in the mRNA levels of c-Myc, mTOR and Notch1 among cells transfected with MECP2 siRNA alone, cells co-transfected with MECP2 siRNA and FBXW7 siRNA, and those transfected with NC-siRNA (Additional file 7: Figure S5A-C). Silencing MECP2 decreased the mRNA levels of Hes1, MMP-2 and MMP-9, while co-transfection with MECP2 siRNA and FBXW7 siRNA rescued these effects ( $P$ $<0.01$, Additional file 7: Figure S5D-F). The protein expression of MECP2 decreased in GC cells after transfection with MECP2 siRNA or MECP2 siRNA + FBXW7 siRNA. Whereas, FBXW7 protein expression increased after transfection with MECP2 siRNA, and MECP2 siRNA + FBXW7 siRNA reversed the effect. Knockdown of MECP2 also decreased the protein expressions of c-Myc, mTOR, Notch1, Hes1, MMP-2 and MMP-9 in whole-cells. Compared with those in cells transfected with MECP2 siRNA alone, the protein expressions of these genes were upregulated in the co-transfection cells. Moreover, the change of nuclear NICD1 protein level was consistent with that of the whole-cell protein level of Notch1 (Fig. 7f). The above findings demonstrated that MECP2 facilitated GC cell migration and invasion through inhibiting FBXW7 transcription, thereby regulating the Notch1/c-Myc/mTOR signaling pathways.

\section{Discussion}

Oncogenesis and tumor progression are the multistep and multifactorial process involving different genes, which is accompanied by alterations in a variety of gene expression patterns that in turn affect cancer cell survival, growth, cycle, apoptosis, migration and invasion regulated by these genes [27]. In recent years, accumulating evidence has certified that MECP2, as a key epigenetic regulator, plays an important oncogene role in several cancer types $[18,28]$. For examle, MECP2 expression is upregulated and promotes tumor progression in breast cancer, lung cancer, cervical cancer and uterine cancer [18]. It enhances oral squamous cell carcinoma and colorectal cancer growth, and facilitates oncogenesis and development of osteosarcoma and neuroblastoma [29, 30]. Silencing MECP2 reduces human prostate 
transformed cell proliferation [31]. Recent research has found that MECP2 regulates cancer cell migration and invasion in glioma and breast cancer $[32,33]$. Our previous studies have proved that MECP2 promotes cell proliferation and cell cycle G1-S transition, and restrains cell apoptosis in liver cancer, gastric cancer and breast cancer [19-22]. By expanding sample, the present study aims to further identify the effect and the molecular mechanism of MECP2 on GC cell migration and invasion. Our results again demonstrate that MECP2 expression is upregulated in primary GC and the high expression is closely related with tumor histology and T stage. The findings suggest that MECP2 may play a key role in GC progression.

Our results demonstrated that high expression of MECP2 in GC significantly promoted cancer cell migration and invasion, while silencing MECP2 remarkably suppressed cell migration and invasion by upregulating the expressions of matrix metalloproteinase-2 (MMP-2) and MMP-9. The process of cancer metastasis is a multi-step biochemical reaction involving many molecular events. An essential step of tumor invasion and metastasis is the degradation of matrix proteins, while the matrix metalloproteinases (MMPs) are the most crucial proteolytic enzymes involved in the process. MMPs facilitate cancer cells accessing vasculature and then the target organs, forming tumor metastasis by degrading the basement membrane and extracellular matrix (ECM) [34]. MMPs can also stimulate cancer cell proliferation and movement to develop metastasis by enhancing the release of growth factors [35]. MMP-2 and MMP-9 can degrade type IV collagen, the major component of basement membranes separating the epithelial cells from the stroma [36, 37], and they have been reported to promote GC cell migration and invasion [38, 39]. Taking these together with the present study, we suggest that MECP2 facilitates GC cell migration and invasion by upregulating MMP-2/9 expression.

Our study identifies FBXW7 as a MECP2-targeting gene. MECP2 binds to the methylated CpG site in the promoter region of FBXW7, resulting in inhibition of FBXW7 transcription. This finding is consistent with that of some previous studies which show that MECP2 functions as a transcriptional repressor by binding to the methylated $\mathrm{CpG}$ sites of gene promoter regions and recruiting corepressors (e.g. histone deacetylases and Sin3A), to restrain the expression of some genes (e.g., MYOD1, FOXF1, BDNF, Cdkl5, RPL11 and RPL5) [17, 20, 21]. FBXW7, a member of the F-box family of proteins, has been characterized as a tumor suppressor gene that plays an important function in cancer cell survival, proliferation, cycle, apoptosis, differentiation, metabolism, tumor metastasis and drug resistance [40, 41]. FBXW7 expression is frequently downregulated in multiple human cancers, such as lung cancer, breast cancer, colorectal cancer, liver cancer, gastric cancer, pancreatic cancer, cervical cancer, prostate cancer, and esophagus cancer [42]. Loss-of-function mutations of FBXW7 are frequently discovered in human cancers, and the total mutation rate is approximately $6 \%$ [43]. Recent studies have shown that FBXW7 suppresses cholangiocarcinoma and colorectal cancer cell migration and invasion [44, 45]. The present study further demonstrates that FBXW7 is frequently downregulated in human GC tissues and FBXW7 exression is correlated with the clinicopathologic features of GC. Overexpression of FBXW7 suppresses GC cell migration and invasion, while silencing FBXW7 promotes cell migration and invasion. Co-transfection with MECP2 siRNA and FBXW7 siRNA rescues the effect of MECP2 knockdown on cell migration and 
invasion. These findings suggest that MECP2 promotes GC cell migration and invasion via suppressing FBXW7 transcription by binding to the methylated $\mathrm{CpG}$ islands of the promoter region of FBXW7.

FBXW7 is a subunit of a SCF-type ubiquitin ligase complex that induces the ubiquitination and proteasomal degradation of oncoproteins, including SREBP1, Cyclin E, c-Jun, c-Myc, mTOR, Notch1, Notch4, MCL-1, KLF5 and MCL-1 [46-49]. This study showed that MECP2 increased the protein expressions of c-Myc, mTOR, and Notch1 by inhibiting FBXW7 transcription and then preventing ubiquitination degradation of oncoproteins. c-Myc is a member of Myc gene family involved in multiple biological processes, such as embryonic development, cell proliferation, cell cycle, apoptosis, differentiation, and protein synthesis [50], and is frequently amplified in many human cancers and promotes cell proliferation, migration and invasion [51]. mTOR acts as a serine/threonine protein kinase that modulates cell proliferation, motility, survival, protein synthesis, autophagy as well as cell cycle progression, and activation of the mTOR pathway promotes cancer cell proliferation, migration and invasion [52]. The Notch signaling pathways regulate cellular differentiation, proliferation and apoptotic events [53]. Notch1-4 are trans-membrane proteins that interact with ligands of the Delta-like and Jagged family. Binding of ligand to its receptor leads to the cleavage of Notch receptor [54] and the Notch1 intracellular domain (NICD1) is generated. NICD1 enters into the nucleus and promotes Hes1, MMP-2 and MMP-9 expressions in some cancers [55-57]. Previous studies have confirmed that Notch1 is upregulated in gastric cancer, liver cancer and ovarian cancer [58-60]. The present study reveals that, by regulating the FBXW7/Notch1 signaling pathway, MECP2 upregulates Hes1, MMP-2 and MMP-9 expressions. MECP2 promotes migration and invasion of GC cells by regulating the Notch1/c-Myc/mTOR signaling pathways through inhibiting FBXW7 transcription (Fig. 8).

\section{Conclusion}

In summary, this study demonstrates that MECP2 promotes GC cell migration and invasion through regulation of the Notch1/c-Myc/mTOR signaling pathways by suppressing the transcription of FBXW7. MECP2 is upregulated and FBXW7 is downregulated in GC tissues, and their levels are closely correlated with the clinicopathological features. The findings indicate that MECP2 may be a novel effective therapeutic target for GC.

\section{Abbreviations}

Aza

5-aza-2'-deoxycytidine; ChIP:Chromatin immunoprecipitation; ChIP-Seq:Chromatin immunoprecipitation sequencing; ECM:Extracellular matrix; FBS:Fetal bovine serum; FBXW7:F-box and WD40 domain protein 7; GC:Gastric cancer; GES-1:Gastric epithelial cell line; IF:Immunofluorescence; IHC:Immunohistochemistry; MBD:Methyl-CpG-binding domain; MECP2:Methyl-CpG-binding protein 2; MMPs:matrix metalloproteinases; MT:mutation type; NICD1:Notch1 intracellular domain; NC-siRNA:Negative control; qRT-PCR:quantitative real-time PCR; SCF:SKP1-CUL1-F-box protein; TCGA:The cancer genome atlas; TRD:Transcriptional repression domain; WT:Wild type 


\section{Declarations}

\section{Availability of data and materials}

The datasets used and/or analyzed during the current study are available from the corresponding author on reasonable request.

\section{Ethics approval and consent to participate}

All the tissue samples have been obtained with the informed consents from patients. The study was reviewed and approved by the Ethical Committee of Xi'an Jiaotong University, Xi'an, China.

\section{Consent for publication}

All authors have agreed to publish this manuscript.

\section{Competing interests}

The authors declare that they have no competing interest.

\section{Funding}

This work was supported by the National Natural Science Foundation of China $(81972603,81772985$, 81702918), the Natural Science Foundation of Shaanxi Province (2020JM-074, 2017JM8112), and Shaanxi Province Key Science and Technology Innovation Team (2020TD-039).

\section{Authors' contributions}

$\mathrm{ZL}, \mathrm{HC}$ and TD conceived and designed the experiments; $\mathrm{LL}$ and $\mathrm{NL}$ ollected clinical data and sample. $\mathrm{ZL}$, WX, YJ, JQ, ZJ and QY performed the experiments. WX and WL performed the statistical analysis. ZL HC and TD wrote and edited the manuscript. HC supervised the work. All authors read and approved the final manuscript.

\section{Acknowledgements}

Not applicable.

\section{References}

1. Bray F, Ferlay J, Soerjomataram I, Siegel RL, Torre LA, Jemal A. Global Cancer Statistics 2018: GLOBOCAN estimates of incidence and mortality worldwide for 36 cancers in 185 countries. CA 
Cancer J Clin. 2018;68(6):394-424.

2. Siegel RL, Miller KD, Jemal A. Cancer statistics, 2016. CA Cancer J Clin. 2016;66(1):7-30.

3. Chen W, Zheng R, Baade PD, Zhang S, Zeng H, Bray F, et al. Cancer statistics in China, 2015. CA Cancer J Clin. 2016;66(2):115-32.

4. Cancer Genome Atlas Research. N. Comprehensive molecular characterization of gastric adenocarcinoma. Nature. 2014;513(7517):202-9.

5. Sun TT, He J, Liang Q, Ren LL, Yan TT, Yu TC, et al. LncRNA GClnc1 promotes gastric carcinogenesis and may act as a modular scaffold of WDR5 and KAT2A complexes to specify the histone modification pattern. Cancer Discov. 2016;6(7):784-801.

6. Zhang JX, He WL, Feng ZH, Chen DL, Gao Y, He Y, et al. A positive feedback loop consisting of C12orf59/NF-KB/CDH11 promotes gastric cancer invasion and metastasis. J Exp Clin Cancer Res. 2019;38(1):164.

7. Vieira JP, Lopes F, Silva-Fernandes A, Sousa MV, Moura S, Sousa S, et al. Variant Rett syndrome in a girl with a pericentric $X$-chromosome inversion leading to epigenetic changes and overexpression of the MECP2 gene. Int J Dev Neurosci. 2015;46:82-7.

8. Chahrour M, Jung SY, Shaw C, Zhou X, Wong ST, Qin J, et al. MeCP2, a key contributor to neurological disease, activates and represses transcription. Science. 2008;320(5880):1224-9.

9. Mellén M, Ayata P, Dewell S, Kriaucionis S, Heintz N. MeCP2 binds to 5hmC enriched within active genes and accessible chromatin in the nervous system. Cell. 2012;151(7):1417-30.

10. Hite KC, Adams VH, Hansen JC. Recent advances in MeCP2 structure and function. Biochem Cell Biol. 2009;87(1):219-27.

11. Sinnett SE, Gray SJ. Recent endeavors in MECP2 gene transfer for gene therapy of Rett syndrome. Discov Med. 2017;24(132):153-9.

12. Gadalla KK, Bailey ME, Cobb SR. MeCP2 and Rett syndrome: reversibility and potential avenues for therapy. Biochem J. 2011;439(1):1-14.

13. Carouge D, Host L, Aunis D, Zwiller J, Anglard P. CDKL5 is a brain MeCP2 target gene regulated by DNA methylation. Neurobiol Dis. 2010;38(3):414-24.

14. McGraw CM, Samaco RC, Zoghbi HY. Adult neural function requires MeCP2. Science. 2011;333(6039):186.

15. Baker SA, Chen L, Wilkins AD, Yu P, Lichtarge O, Zoghbi HY. An AT-hook domain in MeCP2 determines the clinical course of Rett syndrome and related disorders. Cell. 2013;152(5):984-96.

16. Shin J, Ming GL, Song H. By hook or by crook: multifaceted DNA-binding properties of MeCP2. Cell. 2013;152(5):940-42.

17. Gabel HW, Kinde B, Stroud H, Gilbert CS, Harmin DA, Kastan NR, et al. Disruption of DNA-methylationdependent long gene repression in Rett syndrome. Nature. 2015;522(7554):89-93.

18. Neupane M, Clark AP, Landini S, Birkbak NJ, Eklund AC, Lim E, et al. MECP2 is a frequently amplified oncogene with a novel epigenetic mechanism that mimics the role of activated RAS in malignancy. 
Cancer Discov. 2016;6(1):45-58.

19. Zhao LY, Zhang J, Guo B, Yang J, Han J, Zhao XG, et al. MECP2 promotes cell proliferation by activating ERK1/2 and inhibiting p38 activity in human hepatocellular carcinoma HEPG2 cells. Cell Mol Biol (Noisy-le-Grand). 2013;(Suppl. 59):OL1876-81.

20. Tong D, Zhang J, Wang X, Li Q, Liu LY, Yang J, et al. MeCP2 facilitates breast cancer growth via promoting ubiquitination-mediated P53 degradation by inhibiting RPL5/RPL11 transcription. Oncogenesis. 2020;9(5):56.

21. Zhao L, Liu Y, Tong D, Qin Y, Yang J, Xue M, et al. MeCP2 promotes gastric cancer progression through regulating FOXF1/Wnt5a/beta-Catenin and MYOD1/Caspase-3 signaling Pathways. EbioMedicine. 2017;16:87-100.

22. Zhao LY, Tong DD, Xue M, Ma HL, Liu SY, Yang J, et al. MeCP2, a target of miR-638, facilitates gastric cancer cell proliferation through activation of the MEK1/2-ERK1/2 signaling pathway by upregulating GIT1. Oncogenesis. 2017;6(7):e368.

23. Kasowski M, Grubert F, Heffelfinger C, Hariharan M, Asabere A, Waszak SM, et al. Variation in transcription factor binding among humans. Science. 2010;328(5975):232-5.

24. Cheng Y, Li G. Role of the ubiquitin ligase Fbw7 in cancer progression. Cancer Metastasis Rev. 2012;31(1-2):75-87.

25. Mao JH, Kim IJ, Wu D, Climent J, Kang HC, DelRosario R, et al. FBXW7 targets mTOR for degradation and cooperates with PTEN in tumor suppression. Science. 2008;321(5859):1499-502.

26. Welcker M, Clurman BE. FBW7 ubiquitin ligase: a tumour suppressor at the crossroads of cell division, growth and differentiation. Nat Rev Cancer. 2008;8(2):83-93.

27. Chaturvedi R, de Sablet T, Asim M, Piazuelo MB, Barry DP, Verriere TG, et al. Increased Helicobacter pylori-associated gastric cancer risk in the Andean region of Colombia is mediated byspermine oxidase. Oncogene. 2015;34(26):3429-40.

28. Müller HM, Fiegl H, Goebel G, Hubalek MM, Widschwendter A, Müller-Holzner E, et al. MeCP2 and MBD2 expression in human neoplastic and non-neoplastic breast tissue and its association with oestrogen receptor status. Br J Cancer. 2003;89(10):1934-9.

29. Meng G, Lv Y, Dai H, Zhang X, Guo QN. Epigenetic silencing of methyl-CpG-binding protein 2 gene affects proliferation, invasion, migration, and apoptosis of human osteosarcoma cells. Tumour Biol. 2014;35(12):11819-27.

30. Murphy DM, Buckley PG, Das S, Watters KM, Bryan K, Stallings RL. Colocalization of the oncogenic transcription factor MYCN and the DNA methyl binding proteinMeCP2 at genomic sites in neuroblastoma. PLoS One. 2011;6(6):e21436.

31. Babbio F, Castiglioni I, Cassina C, Gariboldi MB, Pistore C, Magnani E, et al. Knock-down of methyl CpG-binding protein 2 (MeCP2) causes alterations in cell proliferation and nuclear lamins expression in mammalian cells. BMC Cell Biol. 2012;11:13-9.

32. Sharma K, Singh J, Frost EE, Pillai PP. MeCP2 overexpression inhibits proliferation, migration and invasion of C6 glioma by modulating ERK signaling and gene expression. Neurosci Lett. 
2018;674:42-8.

33. Liu Y, Jin X, Li Y, Ruan Y, Lu Y, Yang M, et al. DNA methylation of claudin-6 promotes breast cancer cell migration and invasion by recruiting MeCP2 and deacetylating H3Ac and H4Ac. J Exp Clin Cancer Res. 2016;35(1):120.

34. Itoh Y, Nagase H. Matrix metalloproteinases in cancer. Essays Biochem. 2002;38:21-36.

35. Nagase H, Visse R, Murphy G. Structure and function of matrix metalloproteinases and TIMPs. Cardiovasc Res. 2006;69(3):562-73.

36. Komatsu K, Nakanishi Y, Nemoto N, Hori T, Sawada T, Kobayashi M. Expression and quantitative analysis of matrix metalloproteinase-2 and - 9 in human gliomas. Brain Tumor Pathol. 2004;21(3):105-12.

37. Köhrmann A, Kammerer U, Kapp M, Dietl J, Anacker J. Expression of matrix metalloproteinases (MMPs) in primary human breast cancer and breast cancer cell lines: New findings and review of the literature. BMC Cancer. 2009;9:188.

38. Lu S, Zhang Z, Chen M, Li C, Liu L, Li Y. Silibinin inhibits the migration and invasion of human gastric cancer SGC7901 cells by downregulating MMP-2 and MMP-9 expression via the p38MAPK signaling pathway. Oncol Lett. 2017;14(6):7577-82.

39. Pagliara V, Nasso R, Di Donato P, Finore I, Poli A, Masullo M, et al. Lemon peel polyphenol extract reduces interleukin-6-induced cell migration, invasiveness, and matrix metalloproteinase-9/2 expression in human gastric adenocarcinoma MKN-28 and AGS cell lines. Biomolecules. 2019;9(12):833.

40. Zhan $P$, Wang $Y$, Zhao $S$, Liu $C$, Wang $Y$, Wen $M$, et al. FBXW7 negatively regulates ENO1 expression and function in colorectal cancer. Lab Invest. 2015;95(9):995-1004.

41. Wang Z, Inuzuka H, Zhong J, Wan L, Fukushima H, Sarkar FH, et al. Tumor suppressor functions of FBW7 in cancer development and progression. FEBS Lett. 2012;586(10):1409-18.

42. Yeh $\mathrm{CH}$, Bellon M, Nicot C. FBXW7: a critical tumor suppressor of human cancers. Mol Cancer. 2018;17(1):115.

43. Akhoondi S, Sun D, von der Lehr N, Apostolidou S, Klotz K, Maljukova A, et al. FBXW7/hCDC4 is a general tumor suppressor in human cancer. Cancer Res. 2007;67(19):9006-12.

44. Yang H, Lu X, Liu Z, Chen L, Xu Y, Wang Y, et al. FBXW7 suppresses epithelial-mesenchymal transition, stemness and metastatic potential of cholangiocarcinoma cells. Oncotarget. 2015;6(8):6310-25.

45. Wang Y, Liu Y, Lu J, Zhang P, Wang Y, Xu Y, et al. Rapamycin inhibits FBXW7 loss-induced epithelialmesenchymal transition and cancer stem cell-like characteristics in colorectal cancer cells. Biochem Biophys Res Commun. 2013;434(2):352-6.

46. Xiao G, Li Y, Wang M, Li X, Qin S, Sun X, et al. FBXW7 suppresses epithelial-mesenchymal transition and chemo-resistance of non-small-cell lung cancer cells by targeting snai 1 for ubiquitin-dependent degradation. Cell Prolif. 2018;51(5):e12473. 
47. Jin X, Yang C, Fan P, Xiao J, Zhang W, Zhan S, et al. CDK5/FBW7-dependent ubiquitination and degradation of EZH2 inhibits pancreatic cancer cell migration and invasion. J Biol Chem. 2017;292(15):6269-80.

48. Tu K, Zheng X, Yin G, Zan X, Yao Y, Liu Q. Evaluation of Fbxw7 expression and its correlation with expression of SREBP-1 in a mouse model of NAFLD. Mol Med Rep. 2012;6(3):525-30.

49. Inuzuka H, Shaik S, Onoyama I, Gao D, Tseng A, Maser RS, et al. SCF(FBW7) regulates cellular apoptosis by targeting MCL1 for ubiquitylation and destruction. Nature. 2011;471(7336):104-9.

50. Chen BJ, Wu YL, Tanaka Y, Zhang W. Small molecules targeting c-Myc oncogene: Promising anticancer therapeutics. Int J Biol Sci. 2014;10(10):1084-96.

51. Dang CV. MYC on the path to cancer. Cell. 2012;149(1):22-35.

52. Hay N, Sonenberg N. Upstream and downstream of mTOR. Genes Dev. 2004;18(16):1926-45.

53. Artavanis-Tsakonas S, Rand MD, Lake RJ. Notch signaling: Cell fate control and signal integration in development. Science. 1999;284(5415):770-6.

54. Bedogni B, Warneke JA, Nickoloff BJ, Giaccia AJ, Powell MB. Notch1 is an effector of Akt and hypoxia in melanoma development. J Clin Invest. 2008;118(11):3660-70.

55. Minella AC, Clurman BE. Mechanisms of tumor suppression by the SCF(Fbw7). Cell Cycle. 2005;4(10):1356-9.

56. Wang X, Zhang J, Zhou L, Sun W, Zheng ZG, Lu P, et al. Fbxw7 regulates hepatocellular carcinoma migration and invasion via Notch1 signaling pathway. Int J Oncol. 2015;47(1):231-43.

57. Yu B, Wei J, Qian X, Lei D, Ma Q, Liu Y. Notch1 signaling pathway participates in cancer invasion byregulating MMPs in lingual squamous cell carcinoma. Oncol Rep. 2012;27(2):547-52.

58. Sun L, Hui AM, Su Q, Vortmeyer A, Kotliarov Y, Pastorino S, et al. Neuronal and glioma-derived stem cell factor induces angiogenesis within the brain. Cancer Cell. 2006;9(4):287-300.

59. D'Errico M, de Rinaldis E, Blasi MF, Viti V, Falchetti M, Calcagnile A, et al. Genomewide expression profile of sporadic gastric cancers with microsatellite instability. Eur J Cancer. 2009;45(3):461-9.

60. Zhou L, Zhang N, Song W, You N, Li Q, Sun W, et al. The significance of Notch1 compared with Notch3 in high metastasis and poor overall survival in hepatocellular carcinoma. PLoS One. 2013;8(2):e57382.

\section{Figures}




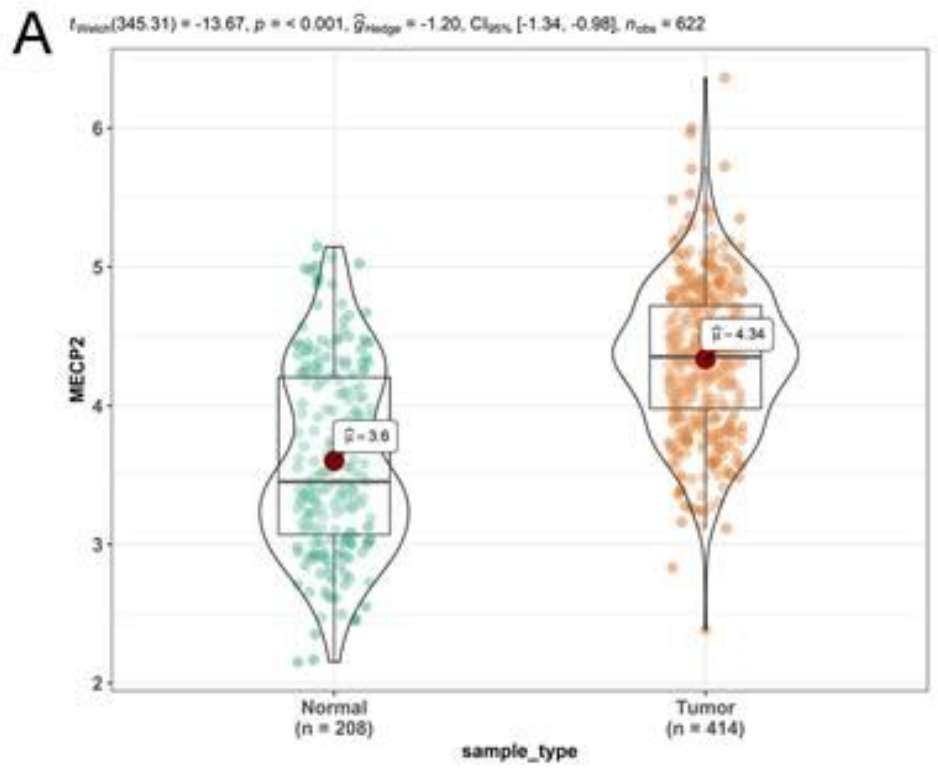

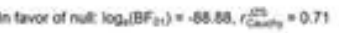

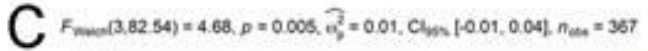
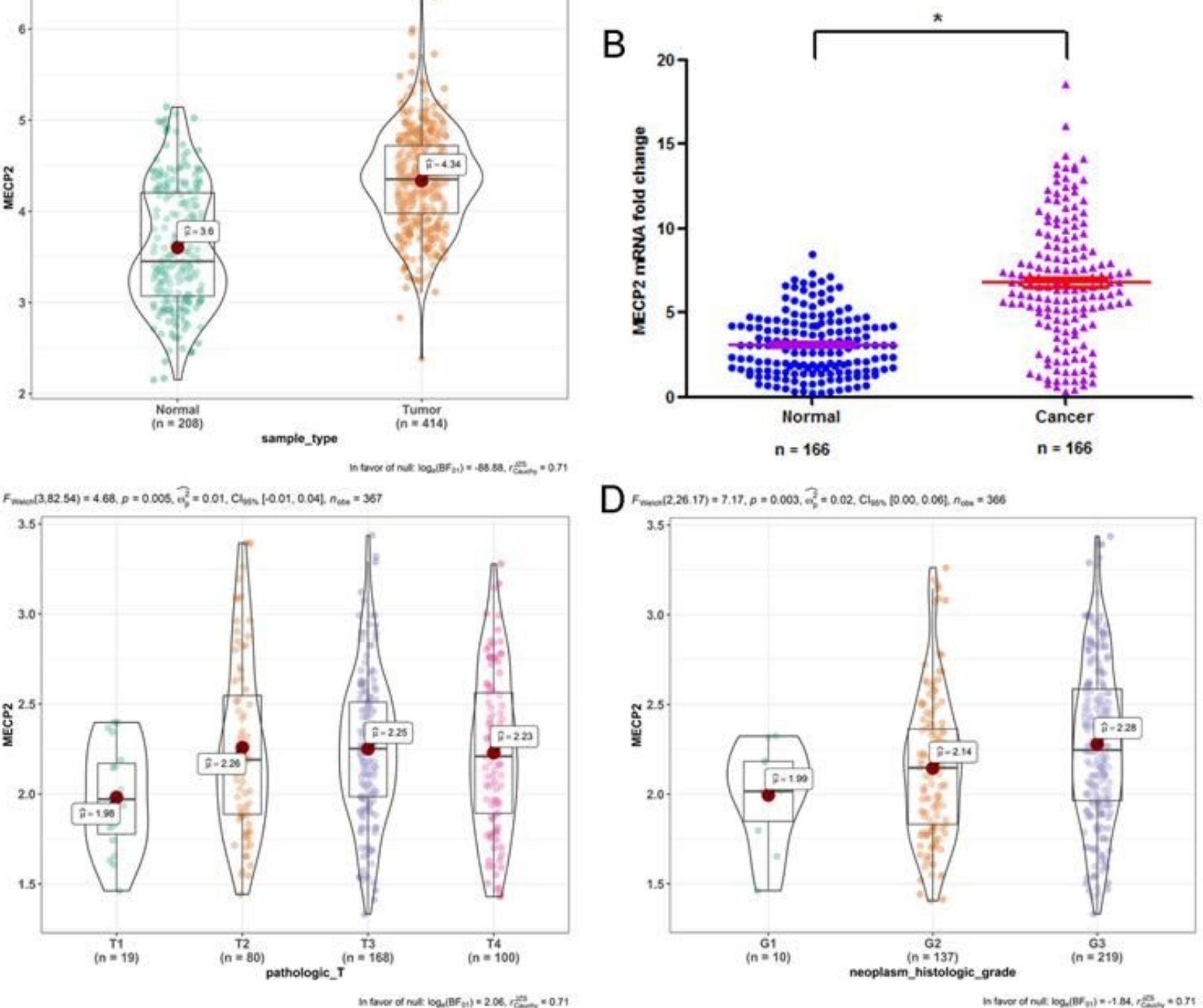

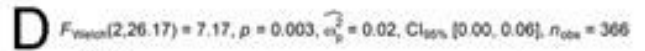

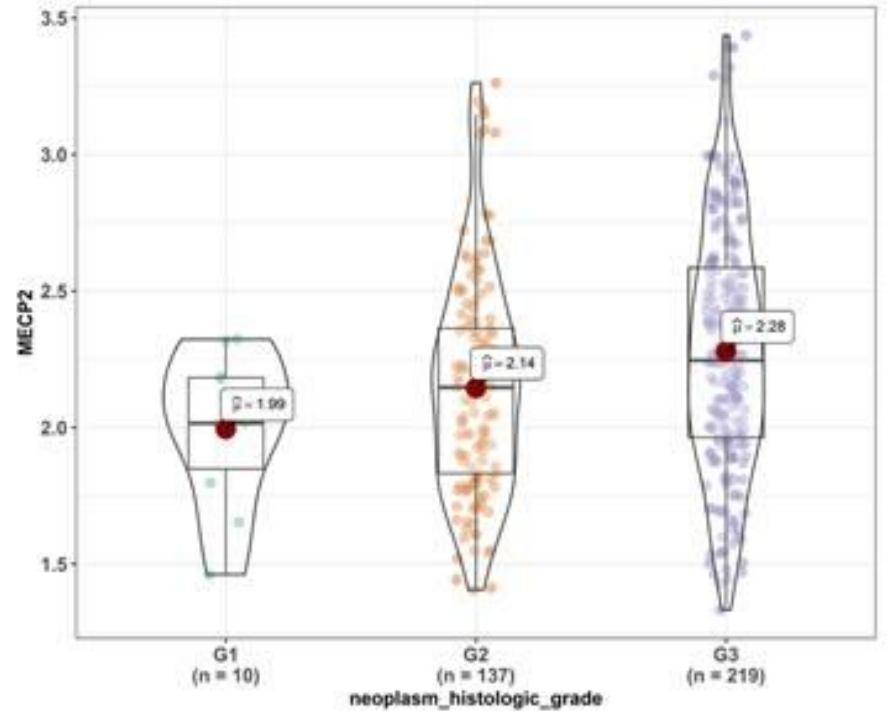

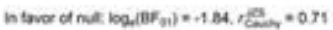

Figure 1

MECP2 is highly expressed in GC and correlated with the clinicopathologic characteristics of GC. a MECP2 expression in tumor and normal tissues using TCGA data. b MECP2 mRNA expression in tumor and normal tissues by qRT-PCR. $n=166$, ${ }^{*}<<0.001$. c Correlation between MECP2 expression and tumor histology based on TCGA data. Data are shown as mean \pm SEM $(P<0.01$, Chi-square test). $d$ Correlation between MECP2 expression and T stage based on TCGA data. Data are shown as mean \pm SEM $(P<0.01$, Chi-square test). 


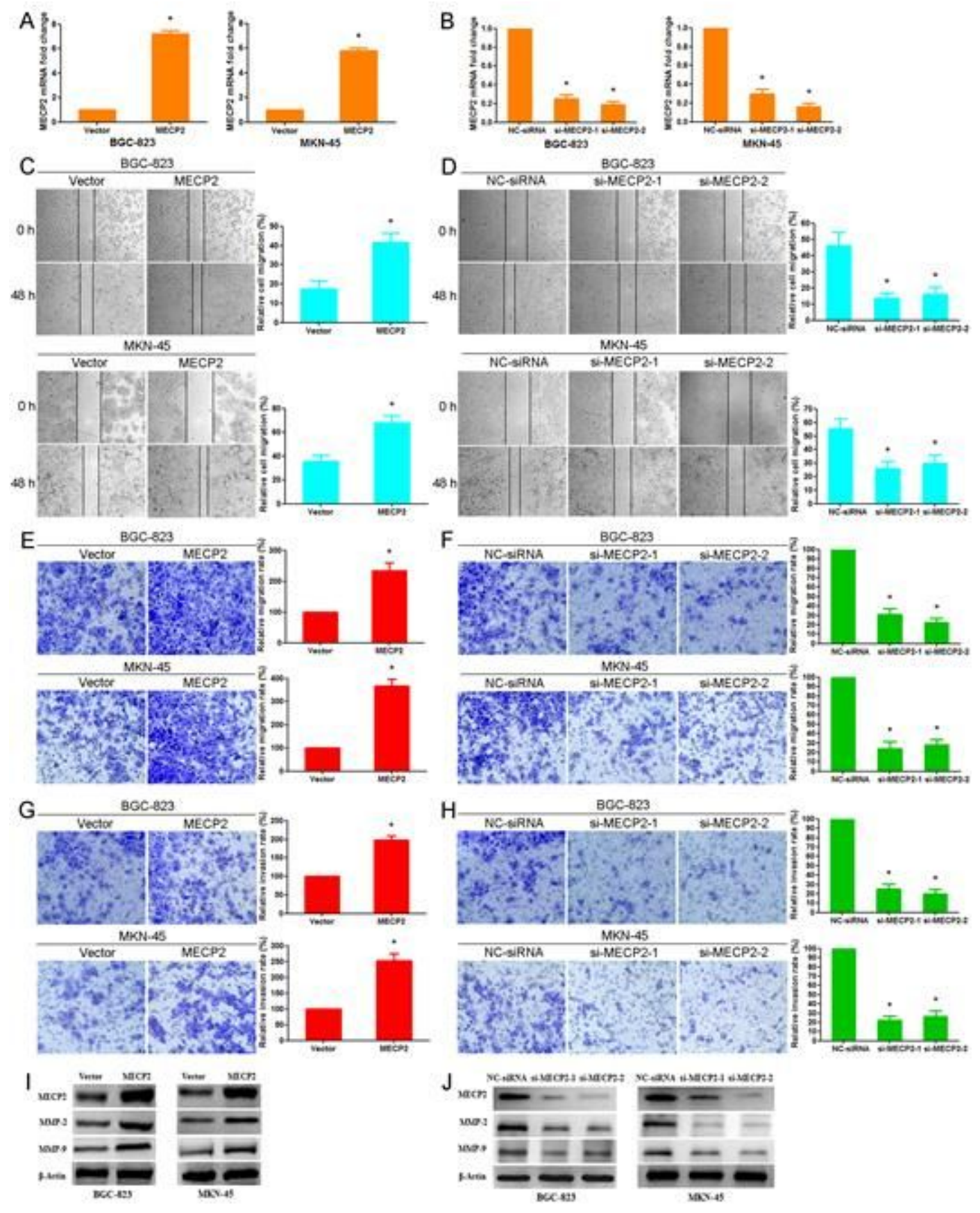

Figure 2

MECP2 promotes GC cell migration and invasion. a MECP2 mRNA expression in BGC-823 and MKN-45 cells $24 \mathrm{~h}$ after transfection with MECP2-overexpressing plasmid. ${ }^{*}<<0.001$. b MECP2 mRNA expression in BGC-823 and MKN-45 cells $24 \mathrm{~h}$ after transfection with MECP2 siRNAs. ${ }^{*} \mathrm{P}<0.001$. c Wound-healing assay. MECP2-overexpressing plasmid promoted cell migration $48 \mathrm{~h}$ after transfection. ${ }^{*} \mathrm{P}<0.01$. $\mathrm{d}$ siMECP2-1 and si-MECP2-2 inhibited cell migration. * $\mathrm{P}<0.01$. e Transwell migration assay. MECP2overexpressing plasmid enhanced cell migration. ${ }^{*} \mathrm{P}<0.01$. $f$ si-MECP2-1 and si-MECP2-2 restrained cell migration. ${ }^{*} \mathrm{P}<0.01 \mathrm{~g}$ Transwell invasion assay. The MECP2-overexpressing plasmid facilitated cell invasion. ${ }^{*} \mathrm{P}<0.01$. $\mathrm{h}$ si-MECP2-1 and si-MECP2-2 suppressed cell invasion. ${ }^{*} \mathrm{P}<0.01$. i MECP2- 
overexpressing plasmid upregulated the protein expressions of MECP2, MMP-2 and MMP-9. j si-MECP2-1 and si-MECP2-2 downregulated the protein expressions of MECP2, MMP-2 and MMP-9.

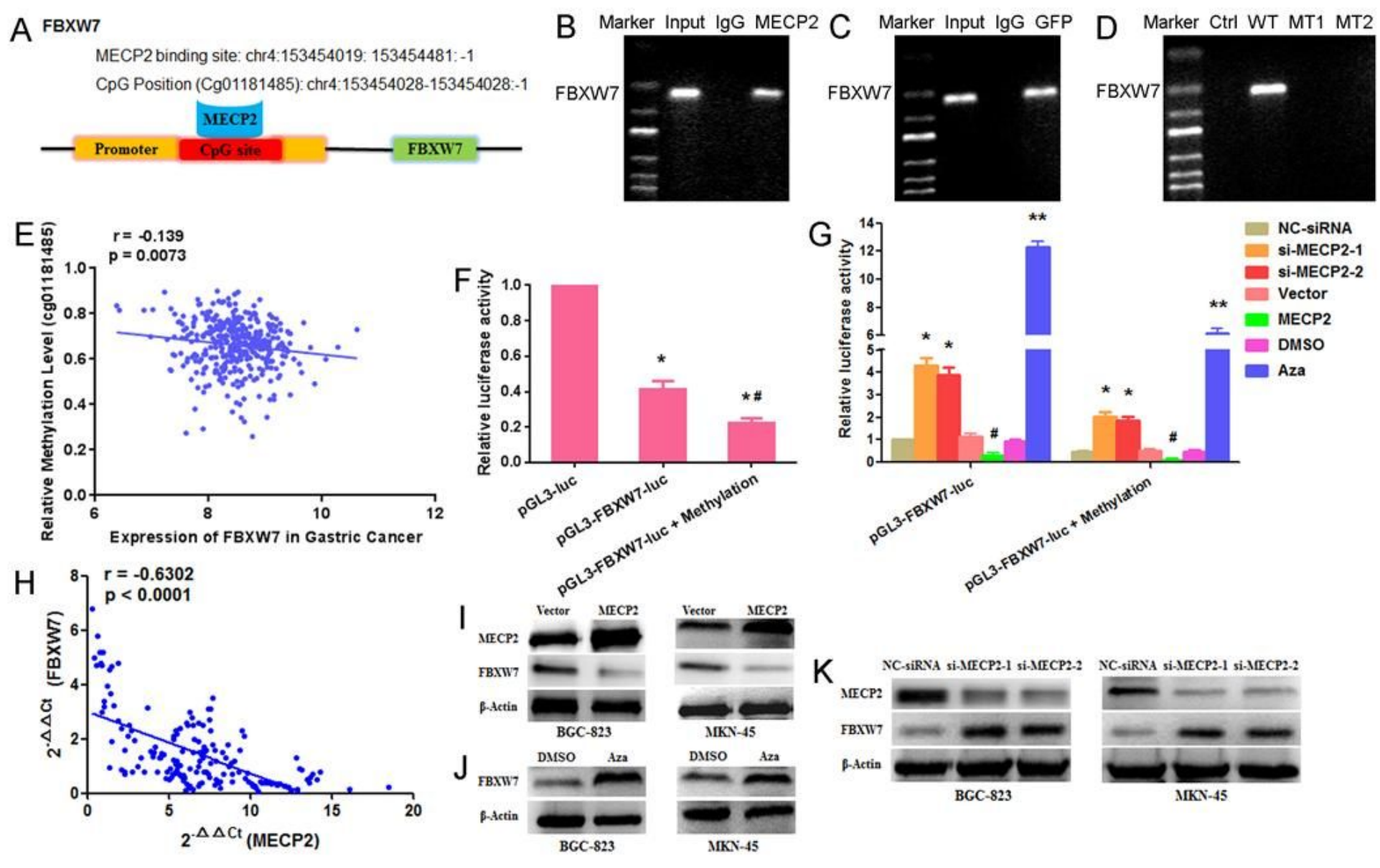

Figure 3

MECP2 inhibits FBXW7 expression in GC cells though binding to the promoter region of FBXW7. a ChIPSeq assay and MethPrimer data. MECP2 bound to the CpG site in the promoter region of FBXW7. b ChIP RT-PCR of FBXW7 with anti-MECP2 antibody. c ChIP RT-PCR of FBXW7 with anti-GFP antibody after transfection with the GFP-MECP2 plasmid. d ChIP RT-PCR of FBXW7 with anti-GFP antibody after transfection with the GFP plasmid (Ctrl), GFP-Mutation type 1 (MT1), GFP-Mutation type 2 (MT2), or GFPMECP2 plasmid (WT). e TCGA data. The methylation level of the CpG site (Cg01181485) was negatively correlated with the FBXW7 expression in GC. $r=-0.139, P=0.0073$, Pearson's correlation. $f$ Luciferase activity in BGC-823 cells examined $48 \mathrm{~h}$ after transfection with pGL3-FBXW7-luc (target sequence of the promoter region of FBXW7) or pGL3-FBXW7-luc + Methylation. Renilla luciferase served as the internal control. *P $<0.01$, as compared with pGL3-luc, \# $\mathrm{P}<0.01$, as compared with pGL3-FBXW7-luc. g Luciferase activity in BGC-823 cells after transfection with pGL3-FBXW7-luc, pGL3-FBXW7-Iuc + Methylation, si-MECP2-1, si-MECP2-2, MECP2-overexpressing plasmid, or methylation inhibitor 5-aza-2'deoxycytidine (Aza). *P $<0.01$, compared with NC-siRNA, $* * P<0.01$, compared with $\mathrm{DMSO}, \# \mathrm{P}<0.01$, compared with vector. $\mathrm{h}$ Negative correlation between MECP2 and FBXW7 levels. The $2-\triangle \Delta \mathrm{Ct}$ values of MECP2 and FBXW7 mRNA were subjected to a Pearson correlation analysis $(r=-0.6302, n=166, P<$ 0.0001). i Western blot. FBXW7 protein expression in BGC-823 and MKN-45 cells after transfection with 
MECP2-overexpressing plasmid. j FBXW7 protein expression after treatment with methylation inhibitor Aza. k FBXW7 protein expression after transfection with si-MECP2-1 and si-MECP2-2.

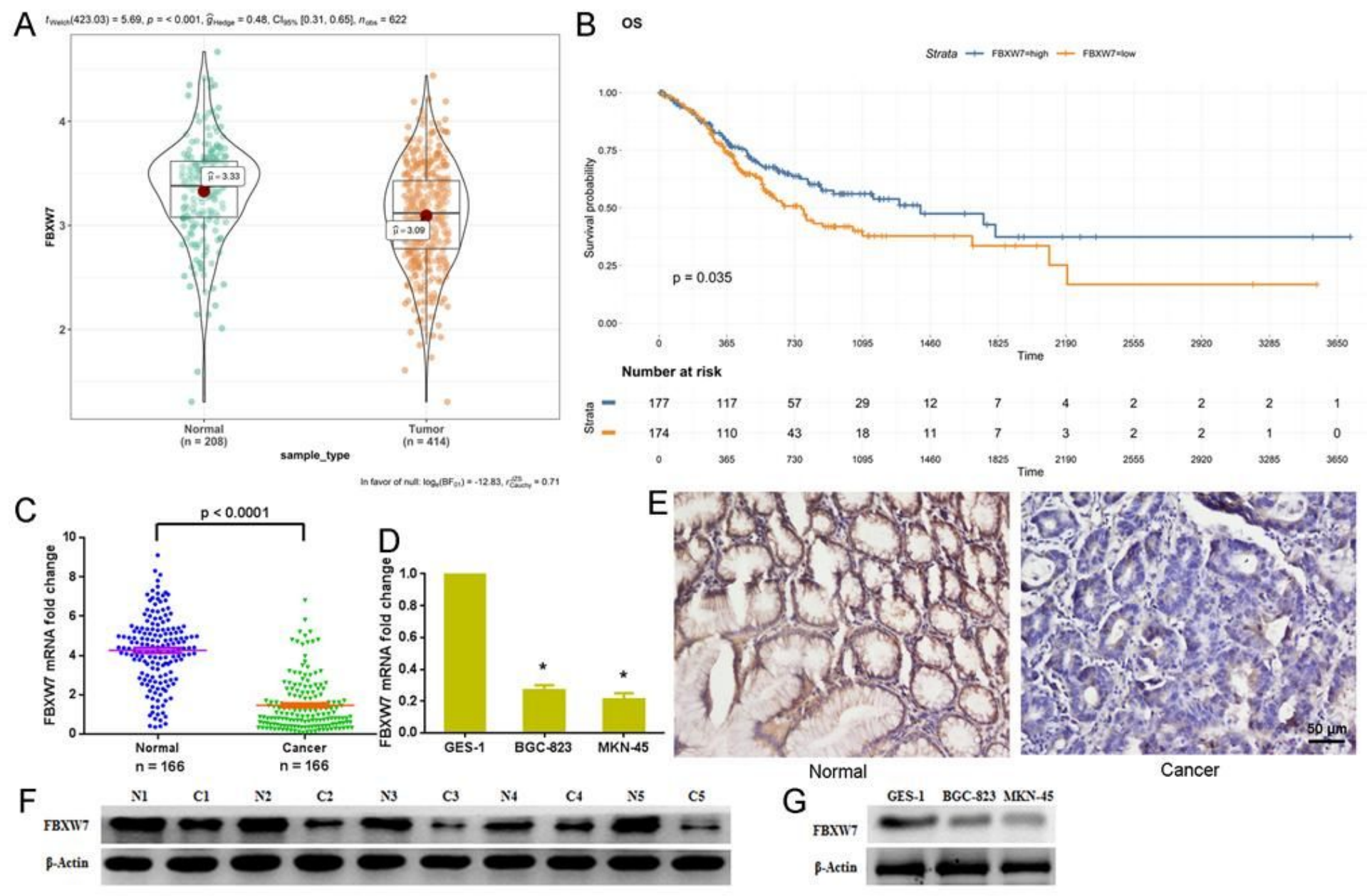

Figure 4

FBXW7 expression is downregulated in human GC tissues and cell lines. a FBXW7 expression in tumor and normal tissues using TCGA data. b Kaplan-Meier curves of overall survival by MeCP2 expression based on TCGA data. c FBXW7 mRNA expression in tumor and normal tissues. $P<0.0001$. d FBXW7 mRNA expression in GC cell lines (BGC-823 and MKN-45) and normal human gastric epithelial cell line (GES-1). ${ }^{*} P<0.001$. e FBXW7 protein expression in tumor and normal tissues by immunohistochemical staining. Scale bars $=50 \mu \mathrm{m}$. f FBXW7 protein expression in GC tissues by Western blot. $\beta$-Actin was used as an internal control. $n=5$. g FBXW7 protein expression in BGC-823 and MKN-45 cells and GES- 1 cells. 

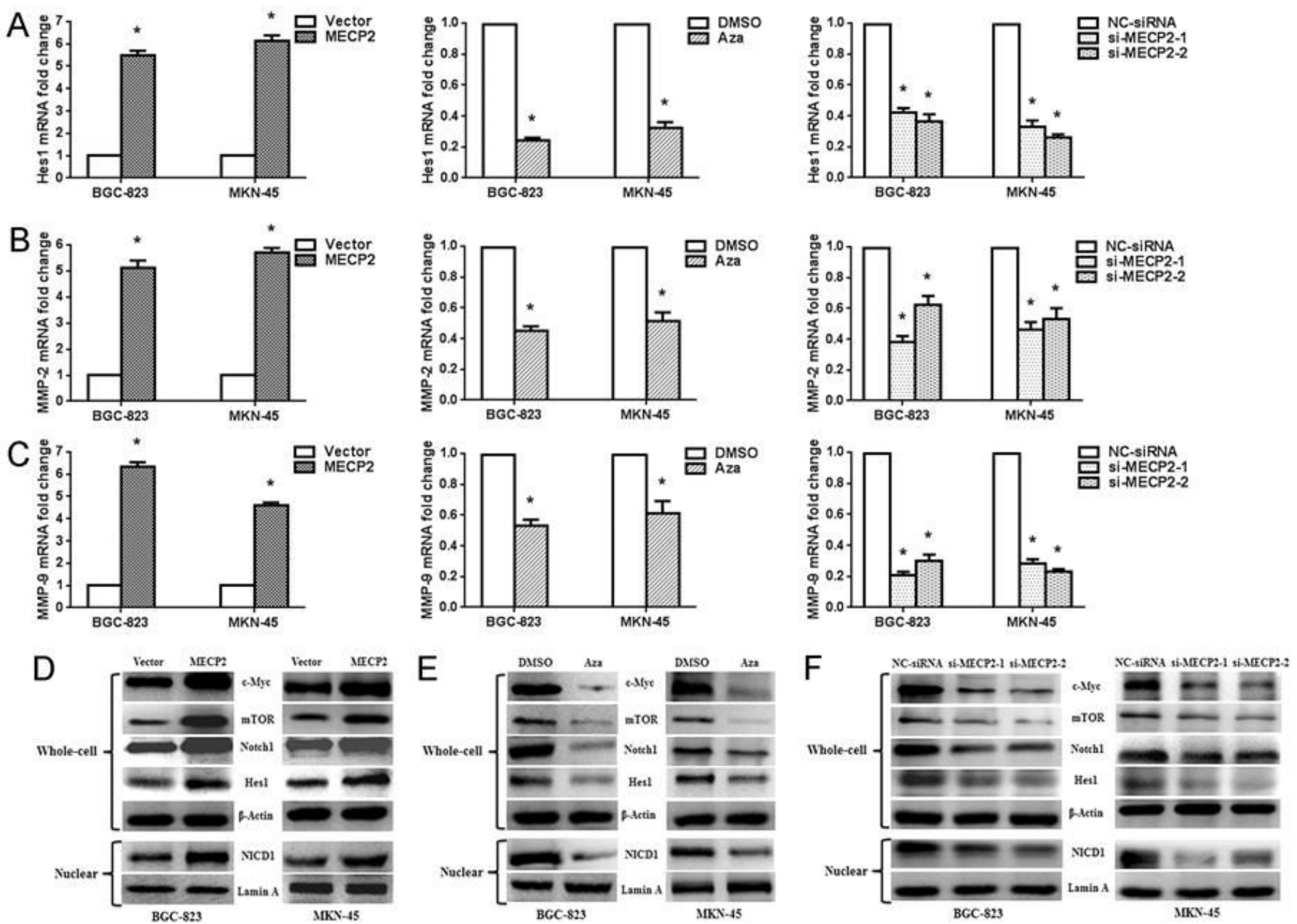

Figure 5

MECP2 regulates the Notch1/c-Myc/mTOR signaling pathways in human GC cells by inhibiting FBXW7 expression. a Hes1 mRNA expression in BGC-823 and MKN-45 cells after transfection with MECP2overexpressing plasmid, methylation inhibitor Aza, MECP2-1, or si-MECP2-2. ${ }^{*} \mathrm{P}<0.001$. b MMP-2 mRNA expression in GC cells after the treatment. ${ }^{*} \mathrm{P}<0.001$. c MMP-9 mRNA expression in $\mathrm{GC}$ cells after the treatment. ${ }^{*} \mathrm{P}<0.001$. $\mathrm{d}$ The related proteins of the Notch1/c-Myc/mTOR signaling pathways after transfection with MECP2-overexpressing plasmid. e The related proteins of the Notch1/c-Myc/mTOR signaling pathways after treatment with methylation inhibitor Aza. $f$ The related proteins of the Notch1/cMyc/mTOR signaling pathways after transfection with MECP2-1 and si-MECP2-2. 

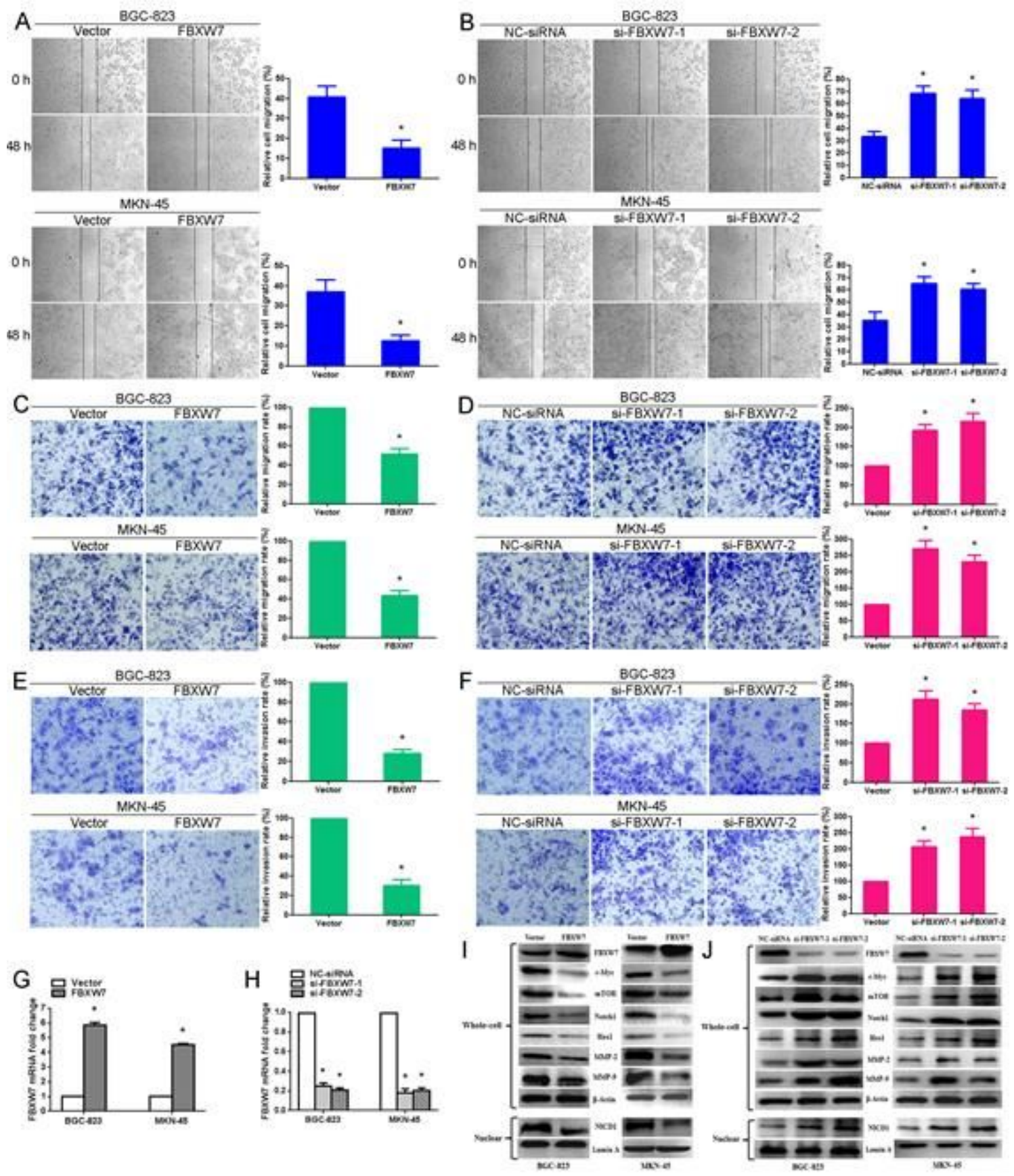

Figure 6

FBXW7 inhibits GC cell migration and invasion. a Wound-healing assay. FBXW7-overexpressing plasmid inhibited BGC-823 and MKN-45 cell migration $48 \mathrm{~h}$ after transfection. ${ }^{*} \mathrm{P}<0.01$. b si-FBXW7-1 and siFBXW7-2 promoted cell migration. ${ }^{*} \mathrm{P}<0.01$. c Transwell migration assay. FBXW7-overexpressing plasmid repressed cell migration. ${ }^{*} P<0.01$. $d$ si-FBXW7-1 and si-FBXW7-2 GC enhanced cell migration. * $P$ $<0.01$. e Ttranswell invasion assay. FBXW7-overexpressing plasmid restrained cell invasion. ${ }^{*} \mathrm{P}<0.01$. $f$ si-FBXW7-1 and si-FBXW7-2 facilitated cell invasion. ${ }^{*} \mathrm{P}<0.01$. g FBXW7 mRNA expression in GC cells 24 $\mathrm{h}$ after transfection with FBXW7-overexpressing plasmid. *P $<0.001$. h FBXW7 mRNA expression in GC cells $24 \mathrm{~h}$ after transfection with FBXW7siRNAs. ${ }^{*} \mathrm{P}<0.001$. i FBXW7-overexpressing plasmid 
downregulated the related proteins of the Notch1/c-Myc/mTOR signaling pathways. j si-FBXW7-1 and siFBXW7-2 upregulated the related proteins of the Notch1/c-Myc/mTOR signaling pathways.
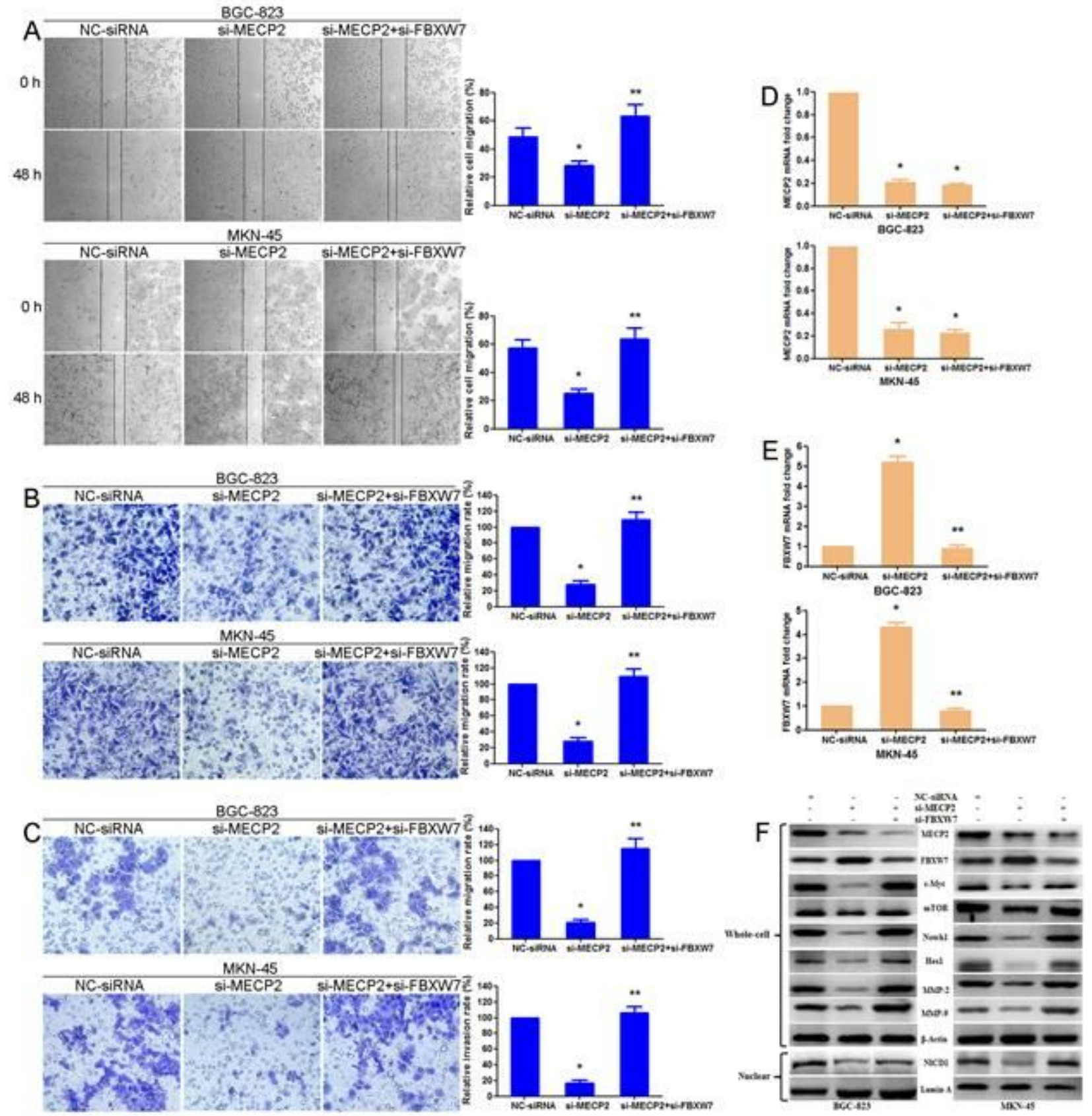

\section{Figure 7}

MECP2 facilitates GC cell migration and invasion by upregulating the Notch1/c-Myc/mTOR signaling pathways through suppressing FBXW7 transcription. BGC-823 and MKN-45 cells were transfected with siMECP2 alone or co-transfected with si-MECP2 and si-FBXW7 a Wound-healing assay. b Transwell migration assay. c Transwell invasion assay. d MECP 2 mRNA expression in cells $24 \mathrm{~h}$ after transfection. $\mathrm{e}$ FBXW7 mRNA expression $24 \mathrm{~h}$ after transfection. $\mathrm{f}$ The related protein expressions of the Notch1/cMyc/mTOR signaling pathways after transfection. ${ }^{*} P<0.01$, as compared with cells transfected with NCsiRNA; $* * P<0.01$, as compared with cells transfected with si-MECP2. 


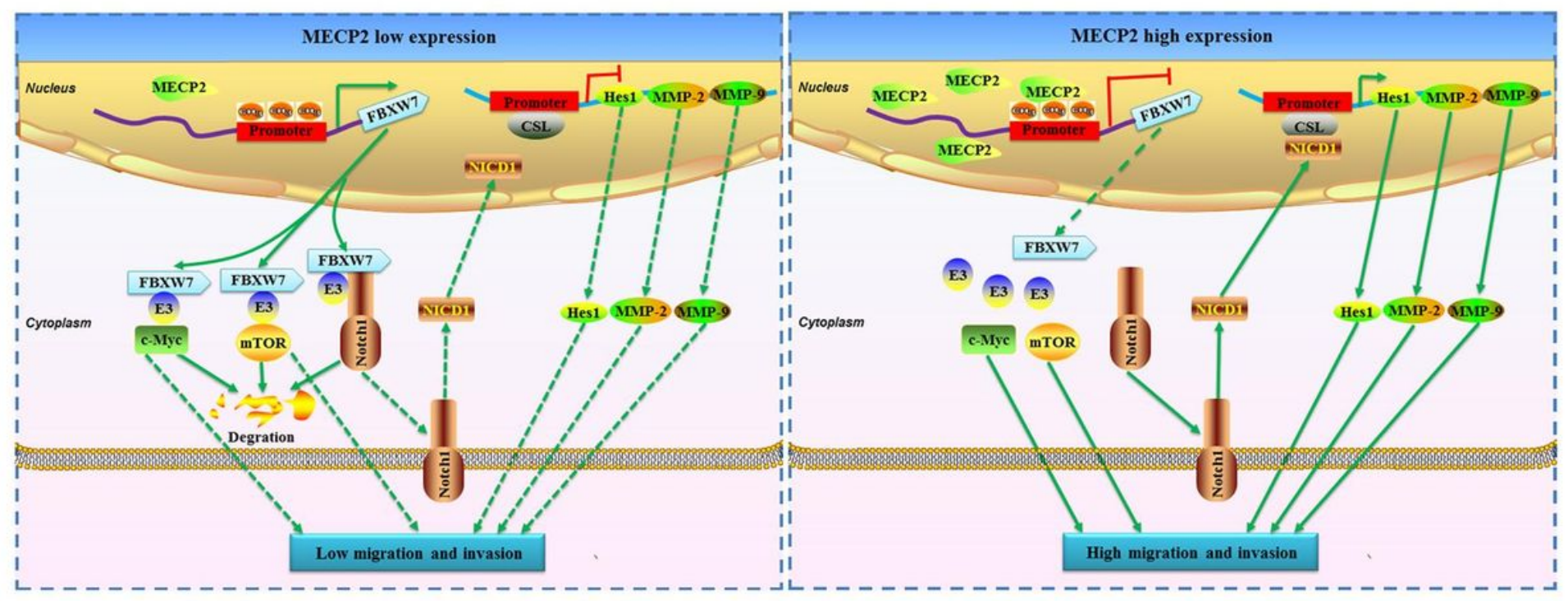

\section{Figure 8}

Proposed model for the effect of MECP2 on GC migration and invasion. MECP2 enhances GC cell migration and invasion through binding to the methylated CpG site in the FBXW7 promoter region and repressing FBXW7 transcription, thereby promoting the Notch1/c-Myc/mTOR signaling pathways.

\section{Supplementary Files}

This is a list of supplementary files associated with this preprint. Click to download.

- PeaksAnnotationaddclassification.xls

- PeaksAnnotationaddclassification.xls

- SupplementaryFigureLegends.docx

- SupplementaryFigureLegends.docx

- SupplementaryFigure5.tif

- SupplementaryFigure5.tif

- SupplementaryFigure4.tif

- SupplementaryFigure4.tif

- SupplementaryFigure3.tif

- SupplementaryFigure3.tif

- SupplementaryFigure2.tif

- SupplementaryFigure2.tif

- SupplementaryFigure1.tif

- SupplementaryFigure1.tif

- SupplementaryTables.doc 
- SupplementaryTables.doc

Page $30 / 30$ 